\title{
Medium temperature solar thermal installation for industrial thermal storage of bituminous
}

\author{
Mircea Bunea, Catherine Hildbrand, Sara Eicher, Alexis Duret, Stéphane Citherlet \\ HEIG-VD, LESBAT, Solar Energy and Building Physics Laboratory \\ Avenue des Sports 20, CH1400 Yverdon-les-Bains, Switzerland
}

\begin{abstract}
In this article, the performance analysis of a medium-temperature solar thermal installation integrated at the Colas Suisse SA industrial site in Yverdon-les-Bains $(\mathrm{CH})$ is presented. The innovative collector system supplies energy for the temperature maintenance of bituminous products and provides part of the heating needs of the administrative building in the industrial site. The initial objective of Colas Suisse SA was to cover approximately $60 \%$ of the thermal energy demand of the site with solar energy. Field measurements provided an insight into the overall operation of the installation during 2015 to 2017. Despite improvement of the system, the performance of the collector field both in terms of energy and financial aspects was found below the expected values. On the other hand, simulations have shown the potential for improvement offered by the current installation. Despite nonoptimal operation of the solar installation, a life cycle impact assessment have indicated interesting potential primary energy savings and greenhouse gas reductions.
\end{abstract}

Keywords: solar thermal, medium temperature collectors; industrial applications; bituminous storage; numerical simulation; measurements

\section{Introduction}

In Europe, the industrial sector accounts for one fourth of the total final energy consumption (Eurostat, 2015) of which more than two-thirds is used for heating purposes (IRENA, 2015). Moreover, 26\% of these process heating concerns low temperatures $\left(<100^{\circ} \mathrm{C}\right)$ while $19 \%$ uses heat between 100 and $400^{\circ} \mathrm{C}$ (Krummenacher \& Muster, 2015). A huge potential exists to provide low to medium temperature processes with heat from existing conventional and advanced collector technologies. It is, therefore, very important to capitalise on this technical potential as today solar energy is reported to cover less than $0.1 \%$ of current industrial heat demand (Mauthner et al., 2016).

In 2012, as part of its emission reduction plan, Colas Suisse, a Swiss subsidiary of Colas International, world leader in construction and maintenance of transport infrastructure, decided to overhaul the heating system of its industrial site in Yverdon-les-.Bains so that $60 \%$ of its thermal energy demand is met through solar energy. A real application of solar thermal integrated in the road construction industry is presented here, where heating needs for bitumen and emulsion storage can be met by temperatures below $200{ }^{\circ} \mathrm{C}$. The thermal and environmental evaluation of this real solar thermal installation is described based on field measurements and numerical simulations.

Bitumen and emulsions are used in road construction, an industry largely relying on fossil fuels, to provide low to medium temperature heat demands. The operating temperature for bitumen emulsion ranges from 60 to $75^{\circ} \mathrm{C}$ while for bitumen it ranges from 160 to $200^{\circ} \mathrm{C}$. These hot products must be stored, transported and used hot to maintain their workability. Conscious of the important potential of solar heating in the industrial context, but also of the economic and environmental issues related to fossil energy use, COLAS Switzerland, has implemented a medium temperature solar thermal system to maintain its bituminous products tanks at the required temperature. The objective was to maximise the share of heat provided by solar heating and optimise the overall energy system. 


\section{System description}

The industrial site located in Yverdon-les-Bains $(\mathrm{CH})$ is composed of a building and the bituminous storage installation situated next to the building. The advantage of this sector is that road construction in Switzerland operates only in the summer season from April to September when solar energy is most available.

A solar collector field was integrated to this industrial site during 2012, but several problems related to wrong hydraulic setups and failures of the control system were identified and therefore important modifications of the installation were performed at the beginning of 2015. The new hydraulic scheme is shown in Fig.1.

Thirty-five solar collectors type C2 (175 $\mathrm{m}^{2}$ aperture area) manufactured by SRB Energy were installed on the site to cover an important share of the thermal energy demand of the industrial site. These collectors are flat plate collectors placed in front of two cylindrical mirrors and requires no solar tracking device. They present a very high efficiency at temperatures above $200{ }^{\circ} \mathrm{C}$ due to the ultra-high vacuum inside the collector $(10-8 \mathrm{mbar}$ at ambient temperature). Thermal oil is used as heat transfer fluid for this system.

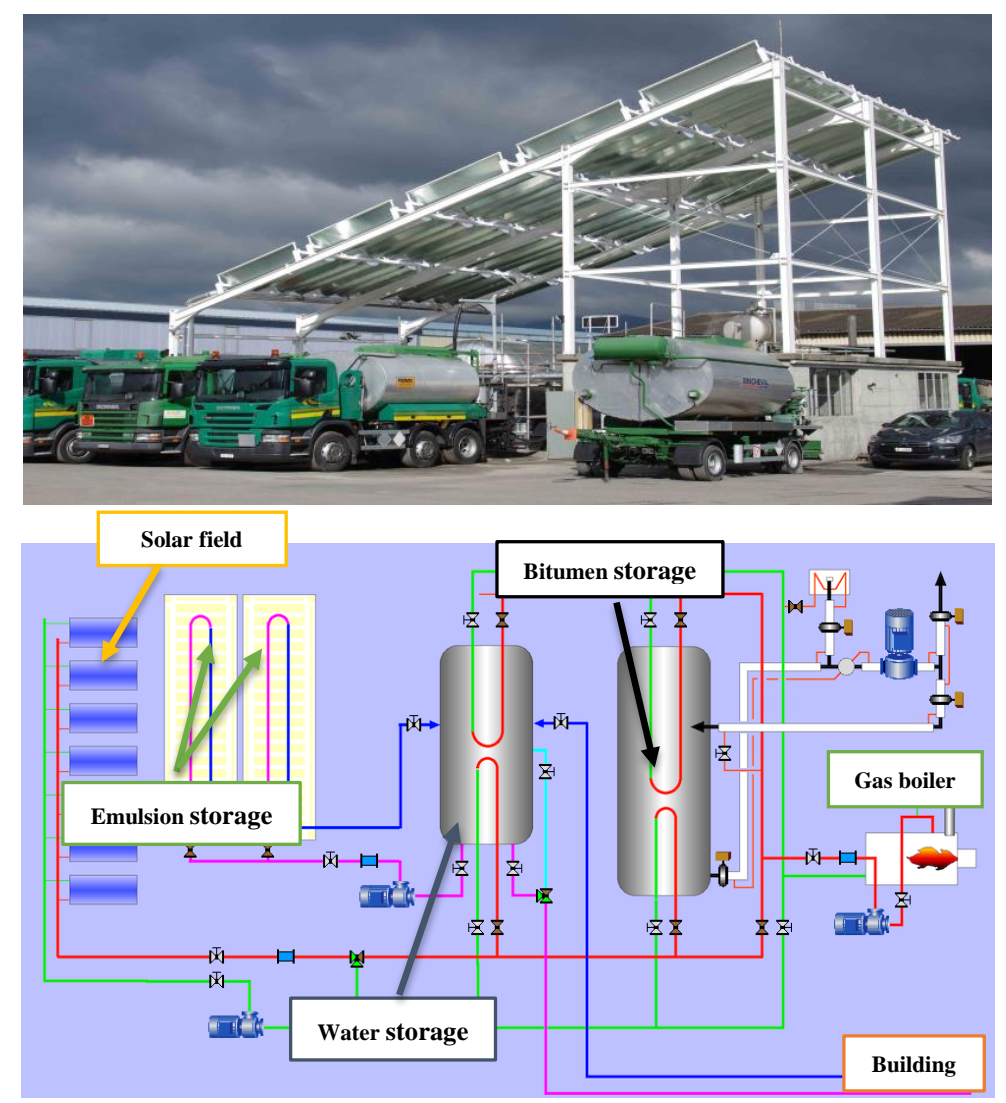

Fig. 1: Picture of the solar installation (up); Simplified representation of the solar thermal installation at COLAS SA after technical modifications (down)

For this particular site, energy is supplied at two different temperature levels:

- $\quad$ Between 50 and $90^{\circ} \mathrm{C}$ for space heating and domestic hot water for the building as well as to keep emulsions (mixture of water and bitumen) at a specific temperature.

- Between 160 and $190^{\circ} \mathrm{C}$ for bitumen storage, also depending on the bitumen's characteristics.

Four storage tanks are also present on the site (see Fig. 1): two $50 \mathrm{~m}^{3}$ emulsion storages, one $70 \mathrm{~m}^{3}$ bitumen storage and one $27 \mathrm{~m}^{3}$ water storage. The latter is used for both building and emulsion heat demand. With the exception of the water storage tank, all other tanks have a variable filling level as the tanks are daily loaded and unloaded depending on the demand.

The solar energy deliver priority is to the water tank in order to lower the operating temperatures of the solar collector field and implicitly maximise collectors' efficiency. If this storage reaches the set point temperature, 
solar energy is delivered to the bitumen storage at higher temperature level. Whenever solar energy is not available, a $250 \mathrm{~kW}$ back-up gas boiler supply the required energy. For further details on this solar thermal installation, please refer to Bunea et al. (2016).

\section{Field measurements results}

Follow-up field measurements and monitoring of the installation have shown the operation of the system as a whole from April 2015 to September 2017.

Bunea et al. (2016) have shown detailed analyse of the system for one year operation. It has been highlighted that the solar yield is strongly influenced by the overall system management. Therefore, it is very important to supply solar energy for low temperature applications (space heat and emulsion needs) in order to optimise collector's efficiency.

Measurement of the collectors' efficiency have shown an important discrepancy between the accredited test values and the measured ones, see Fig. 2. Differences observed for the optical efficiency (15\%) can be partly explained by eventual dust or bituminous particles deposits on the collector glasses and mirrors. A thermographic analysis of the solar collectors' field has further suggested that the thermal insulation of the UHV is also not fully ensured for some collectors. Consequently, global efficiency of the collector field is compromised. Differences may also appear as the curve from the certification tests (blue) is given for a fixed irradiation of $800 \mathrm{~W} / \mathrm{m}^{2}$ while the measurements are done considering real time fluctuating conditions.

For high operating temperatures, the difference with the theoretical efficiency of the collector exceeds $50 \%$. This highlights the fact that extrapolation of certified tests results beyond $100^{\circ} \mathrm{C}$ is not appropriate for evaluating these solar thermal collectors.

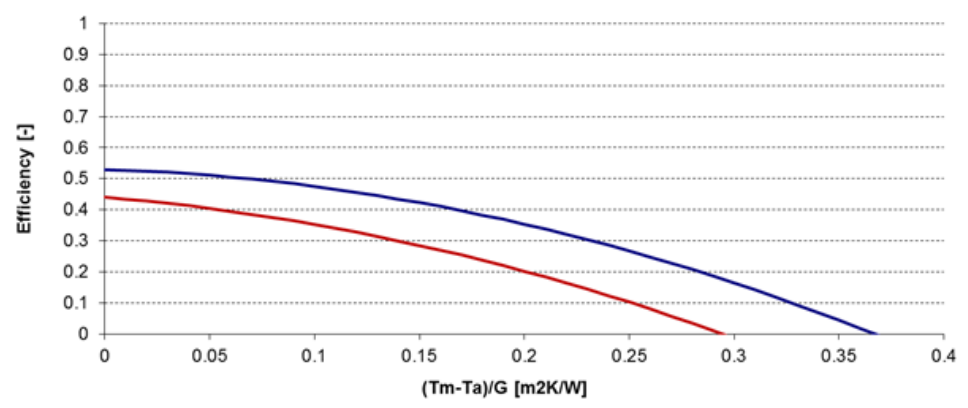

—SRB UHV type $c 2$ certified $\quad$-SRB UHV type $c 2$ validated

Fig. 2: Comparison of SRB type C2 collector efficiency from certification testing and field measurements

This section presents the evolution of the system operation during three full bitumen seasons from April 2015 to September 2017. Globally it can be seen that the bitumen loaded and unloaded activites in the tank are constantly decreasing every year, which means less activity for Colas and implicitly lower energy consumption, see Fig. 3. The reason for this low activity is the large number of roads built and/or repaired before 2015 that imply no important road work after that. This points out the strong irregularity of the consumption profile over the different years.

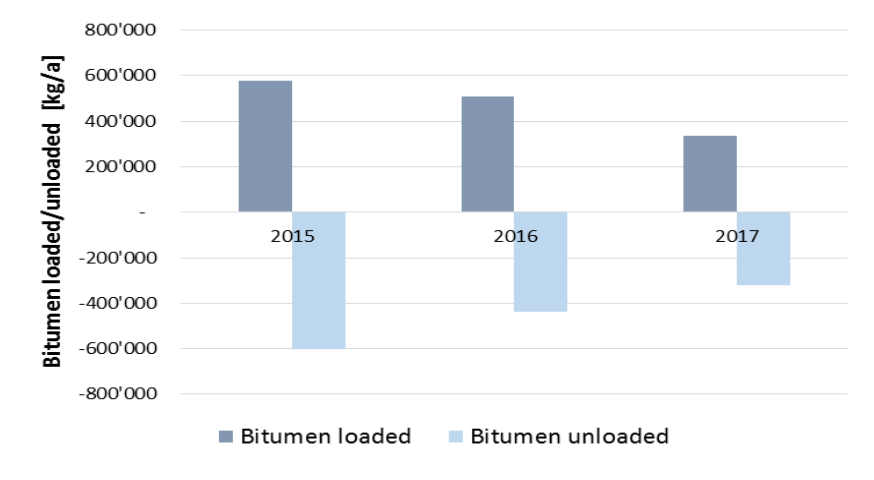

Fig. 3: Quantity of bitumen loaded and unloaded in the bitumen tank 
This low activity has also an impact in the solar field production due to a decreasing energy demand, but Fig. 4 shows that the solar yield is relatively constant over the years. This happens because the control management gives priority to the low temperature heat demand and therefore most solar energy is delivered to the water storage with only 10 to $21 \%$ of solar energy delivered to the bitumen storage yearly.

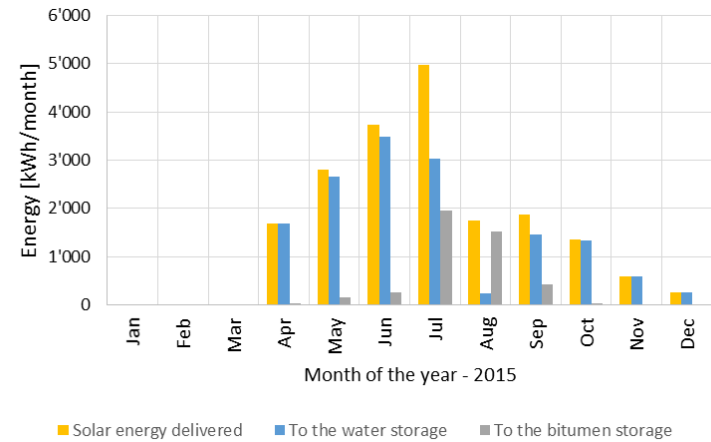

a)

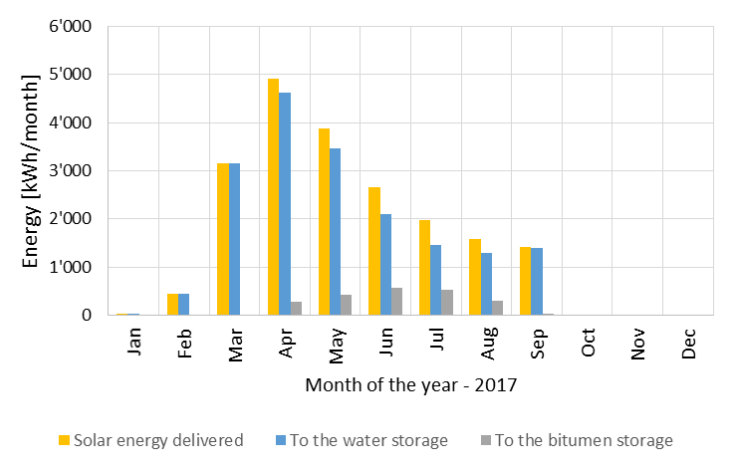

c)

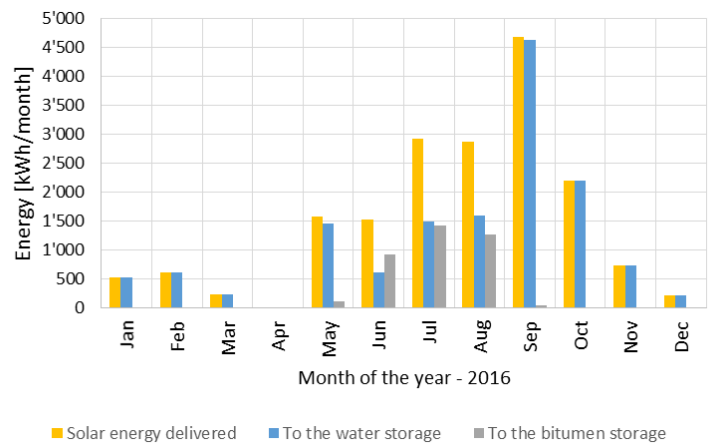

b)

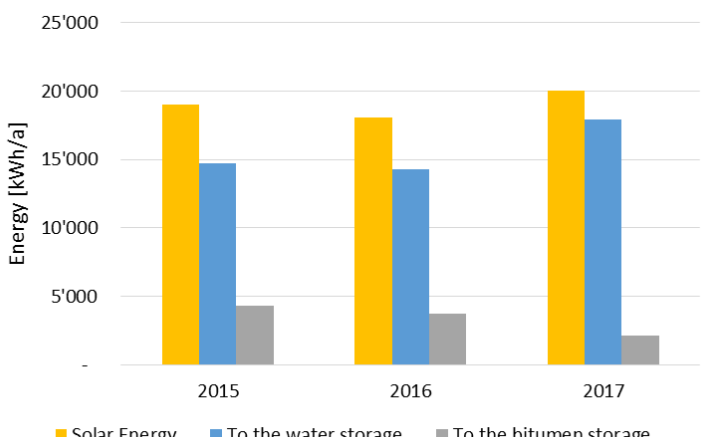

d)

Fig. 4: Solar energy delivered to the system. a) in 2015, b) in 2016, c) in 2017 and d) yearly

Fig. 4 also indicates that the solar production profile over one year is also very different not only because weather conditions vary, but particularly because of the high irregularity of the demand profile due to the different construction sites and their specific demand for a given year. The control strategy and the eventual breakdowns occurred at different points of the system can also affect considerably the solar efficiency. For example, a breakdown in the emulsion system in August 2015 caused a drastic decrease in solar production, with sensibly the same weather conditions as July. Under this configuration, solar collectors delivered energy mainly to the bitumen storage, operating at higher temperatures, which greatly reduced the efficiency of the solar thermal system. Another breakdown of the system forced the operator to completely shut off the solar pump from March to early May 2016, reducing to 0 the solar energy delivered to the system during this period.

The year 2017 was characterised by the non-utilisation of the emulsion products stored on site, a strategic decision taken by Colas. Consequently, the reduction of the low temperature energy demand during the bitumen season decreased the solar yield during the summer of 2017. This points out, once again, the tremendous influence of the heat demand on the heat supply for low exergy production devices like solar thermal collectors.

As the solar collectors supply energy to the water tank, their performance changes from that when energy is delivered to the bitumen tank due to their different operating temperature. To illustrate this Fig. 5 shows a comparison of the performances under these two supply configurations against the daily radiation. 


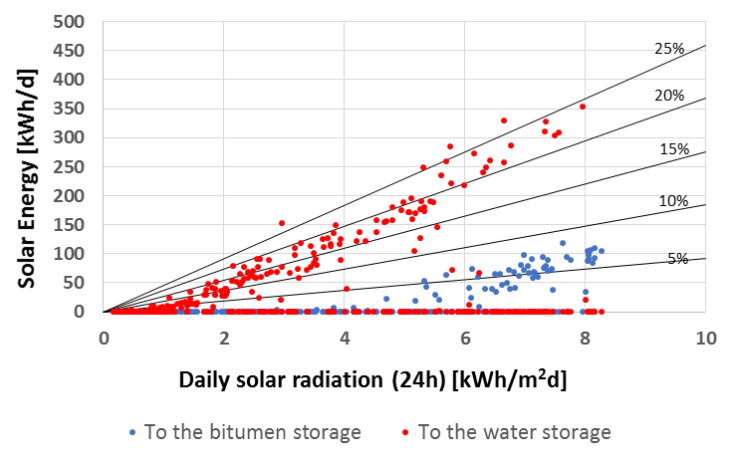

a)

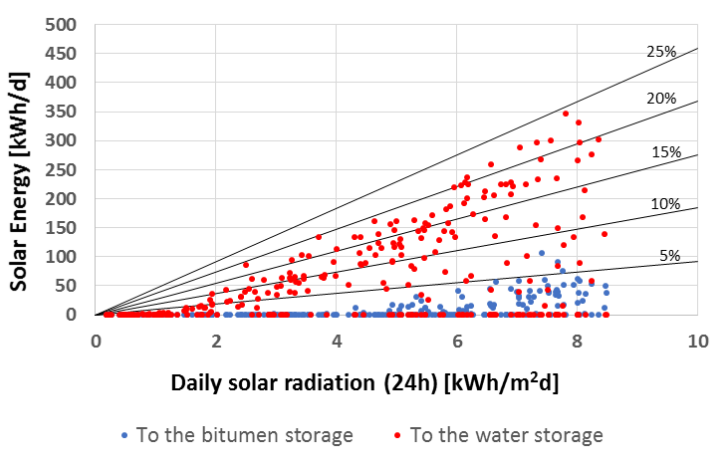

b)

Fig. 5: Performance distribution of solar collectors. a) for 2016, b) for 2017

Overall, the performance is below expectations under all operating modes. The distributions indicate that the collectors' performance decrease when charging the bitumen tank (blue dots) with values below 10\%. However, when delivering energy to the water tank (red dots) the performance is improved and could reach $25 \%$. In this case, scattering of the data is more pronounced because of the different utilisation modes of the water tank. Data points at $0 \%$ performance correspond to days where the collectors' pump was operating but no heat was delivered to any of the two tanks. This happens because the operating temperature is lower than the storage tanks temperature. Consequently, $35 \%$ of the total energy used by the solar pump was spent for circulation only, clearly revealing a controller malfunction.

An evolution of collector's efficiency can be observed between the two years of operation and this for both operation modes. In 2016, when delivering energy to the water storage, the collector field operated with a mean efficiency around 20\%, while in 2017 the efficiency decreases at around $15 \%$ for this application. Additionally, when delivering energy to the bitumen tank a decrease from 7\% in 2016 to less than 5\% in 2017 is observed. The main reason for this decrease could be related to the vacuum loss inside the solar collectors, increasing the thermal losses of the collector itself.

Another point highlighted during this project was the important energy consumption needed for heating a few meters of hydraulic ducts used for loading and unloading the bitumen tank. These pipes are constantly maintained at $220^{\circ} \mathrm{C}$ to enable sufficient fluidity and proper workability of the bitumen. Fig. 6 shows that in the first years of operation the energy consumed for this circuit was in the range of the energy used for maintaining several tons of bitumen in temperature. Advised by LESBAT, different measures were applied in 2017 by Colas, acting both on the control management and on the thermal insulation of the pipes. These measures enabled a reduction of this consumption to less than $50 \%$ of the energy needed for bitumen maintain in temperature during 2017.

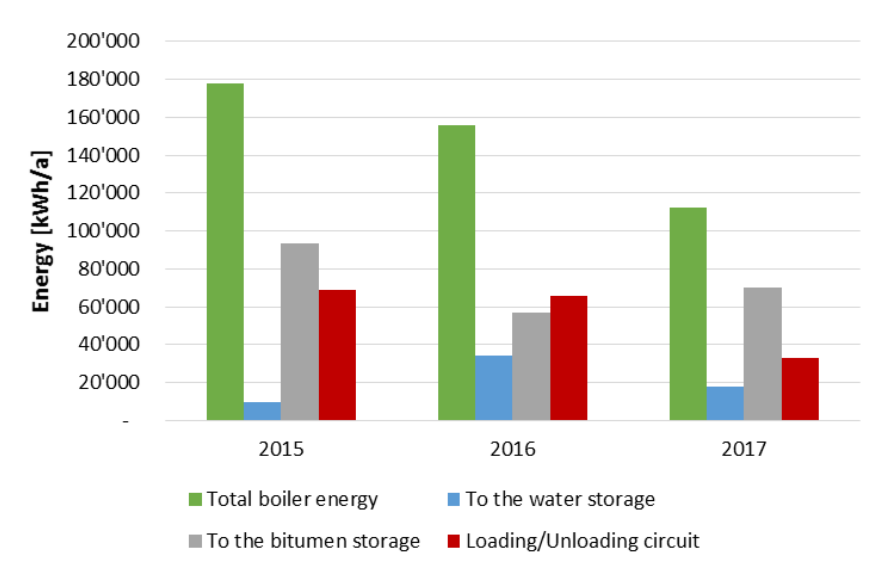

Fig. 6: Breakdown of energy delivered by the gas boiler 


\section{Numerical simulation results}

Numerical simulations of the installation performed with Polysun, showed the potential for energy management improvement. A parametric study was carried out to study the effect of different operating and design parameters on the overall installation performance. For example, improving emulsions processing with heat provided by solar energy or gas boiler through the water storage could result in $25 \%$ increase of the annual solar production, see Fig.7. In addition, improvements of the collector efficiency could double the current annual solar production.

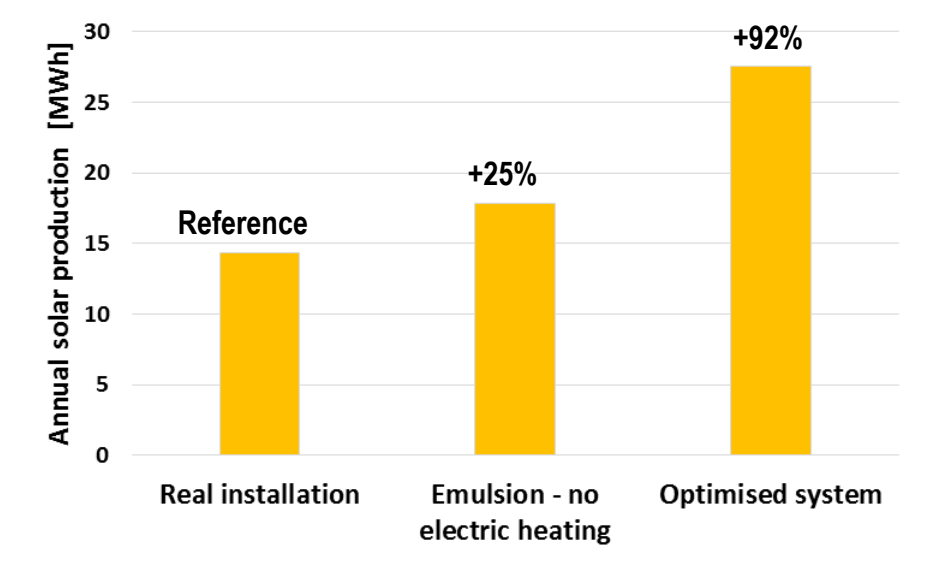

Fig. 7: Simulated annual solar production through system optimisation

Other simulations to predict the effect of other parameters such as collector surface area or type, on the annual solar production, demonstrate that the installation as a whole could absorb much more solar energy, see Fig. 8.

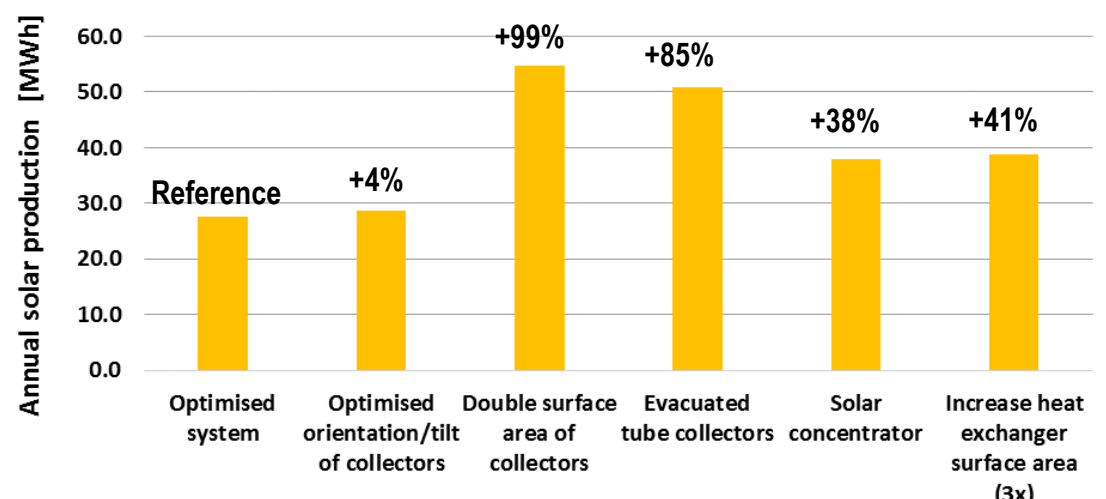

(3x)

Fig. 8: Comparison of the predicted annual solar production for different configurations

It can be seen that optimisation of the orientation and tilt of current installed collectors do not significantly increase the annual solar production. However, doubling the collector surface area could potentially double this production mainly because the share of solar heating in the reference, optimised installation, is still minimal. Interesting to notice is the $85 \%$ increase in solar production by replacing the SRB collectors with evacuated tube collectors with equivalent aperture area. This type of collector is, nevertheless, not able to provide heat in the temperature range of bitumen processing, the primary reason for considering solar thermal integration in this industrial process.

The use of solar concentrators also indicates an increase in solar production (up to 38\%). In all these simulations, most of the solar energy harvested is supplied to the water tank but solar heating of the bitumen tank presents a better efficiency when compared with the two other collectors. Disadvantages of this technology are the additional investment and operating costs as well as the increase of the effective space occupied by the system. Finally, following observations that the heat exchanger performance in the tanks was not optimal, simulations were used to investigate the effect of varying the heat exchanger surface area on the annual solar production. As the heat exchanger area is increased the solar production increases considerably, demonstrating that the actual heat exchange surface is largely undersized. 


\section{Financial analysis}

In order to estimate the financial efficiency of the solar system installed by Colas in Yverdon-les-Bains and to evaluate the solar energy cost, a short financial analysis was conducted taking into consideration costs given by the industrial partner and measurements operated on site.

The total system investment cost was evaluated at $365 \mathrm{kCHF}$ (1'984 CHF/m² - aperture area). Data and assumptions made for cost evaluations are given in Table 1 and the breakdown of these costs is given in Fig. 9 a). This shows the low share $(39 \%)$ of the solar collector field compared to the system integration $(60 \%)$. The imposing stainless steel structure designed to hold the collector field was very costly and significantly increases the overall system cost. Moreover, this structure required some modifications after its installation in order to meet local standards in terms of aesthetics.

Tab. 1 Financial analysis - Data and assumptions

\begin{tabular}{|c|c|}
\hline Data/assumption & Value \\
\hline Investment cost & $380^{\prime} 200 \mathrm{CHF}$ \\
\hline Subsidies & $15^{\prime} 000 \mathrm{CHF}$ \\
\hline Operation \& Maintenance costs & $8^{\prime} 000 \mathrm{CHF} / \mathrm{y}$ \\
\hline System life span & 20 years \\
\hline Interest rate & $3 \%$ \\
\hline Annuity factor & $6.72 \%$ \\
\hline
\end{tabular}

The price of this system is therefore much higher than conventional prices for solar thermal installations (Mauthner et al., 2016), but it must be considered that this was a pilot project, first of its kind, and almost everything was custom made. Of course, there is a lot of room for cost optimisation, starting with canceling the holding structure and improving the maintenance of the system. The solar collector manufacturer should also try to reduce the collector costs for a better competitiveness on the market.

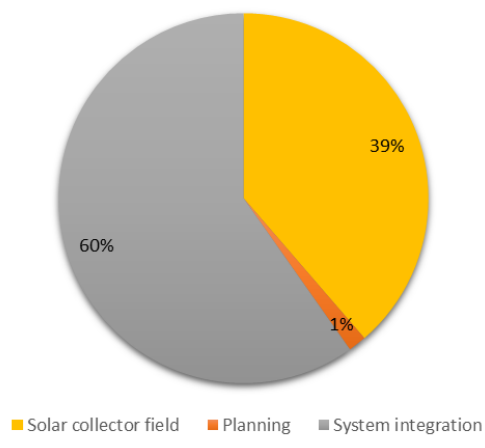

a)

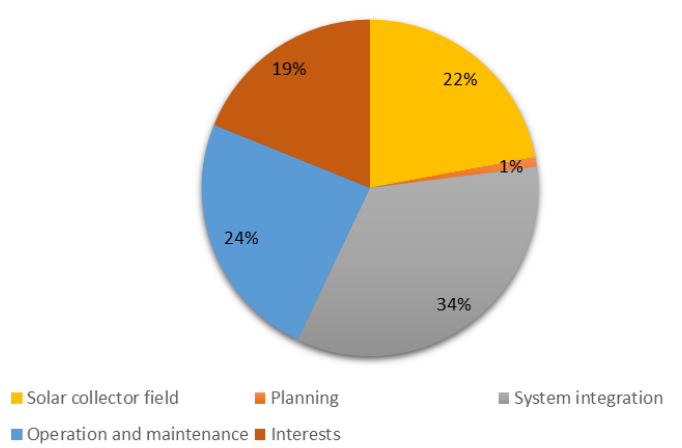

b)

Fig. 9: Solar system cost breakdown for the Colas industrial site: a) Investment costs, b) costs over the life span

Energy costs were evaluated using the uniform capital recovery method. By adding the operating and maintenance costs to the total investment cost and taking into account the interest rate, the cost of solar energy is obtained and can be compared to other conventional energy sources. These costs were calculated for both the real operation of the system and for an optimised system as presented in the previous section (numerical simulations).

The results give $1.80 \mathrm{CHF} / \mathrm{kWh}$ in the actual operation mode which is far from being satisfying and the reason for this high energy cost is mainly the investment cost, but also the non-optimal system operation. With an optimised system, the price is reduced to $1.18 \mathrm{CHF} / \mathrm{kWh}$ which is much better, but it is still 10 times higher than the actual price of natural gas paid on site. Therefore, an estimation of this price was calculated with an optimised investment cost, not taking into account the holding structure, and reducing by $50 \%$ the operating and maintenance spending. The result gives $0.47 \mathrm{CHF} / \mathrm{kWh}$, which can be acceptable for this kind of pilot project.

Furthermore, if evacuated tube collectors were used instead of the SRB collectors the price could be reduced to $0.11 \mathrm{CHF} / \mathrm{kWh}$ considering an investment cost of $500 \mathrm{CHF} / \mathrm{m}^{2}$. This price could be easily reached for important collector fields, without special holding structure (Mauthner et al., 2016). In this case, solar energy can be 
considered as competitive with any other energy source, but once again, this system could only provide low temperature level energy demand and could not supply the bitumen storage tank.

\section{Life cycle impact assessment results}

A life cycle impact assessment was carried out to quantify the environmental impacts of the solar installation. Methodology details and indicators considered in this study have been described elsewhere; please refer to Bunea et al. 2016 for additional information.

\subsection{Impact of the solar installation}

The analysis aims to estimate the environmental impact of the solar installation (solar collectors, pipework components and supporting structure) to compare to the potential energy savings it could possibly bring. Energy consumption of the installation was defined based on monthly simulations. Fig. 10 shows that impacts from the supporting structure are the major contributor from the overall solar installation.

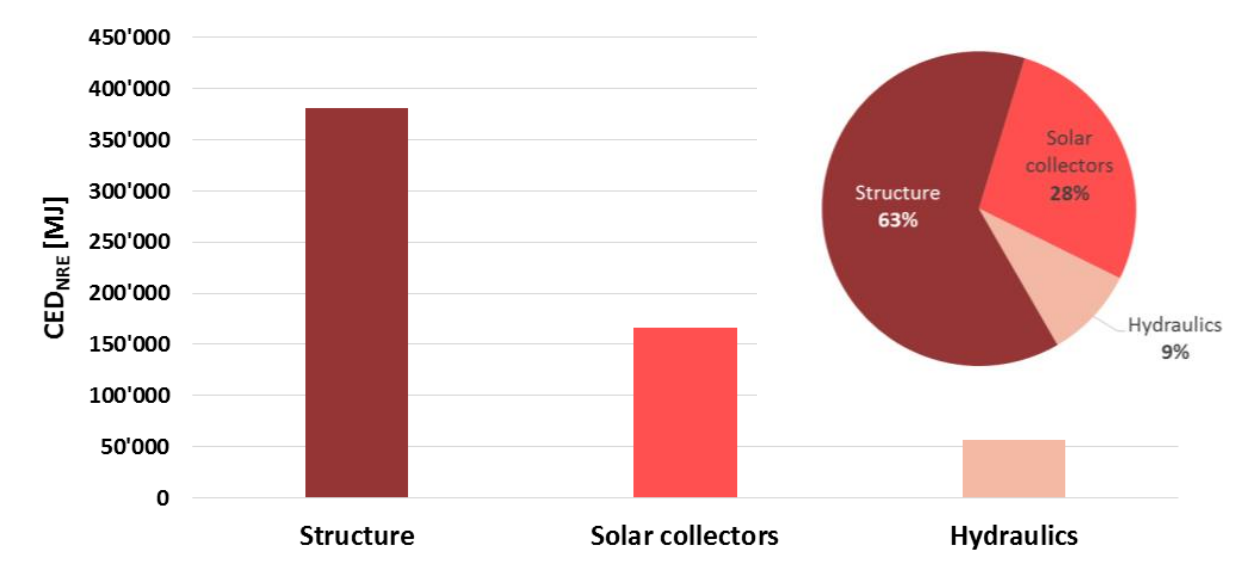

Fig. 10: Calculated environmental impacts of the solar installation, $\mathrm{CED}_{\mathrm{NRE}}$

\subsection{Potential environmental savings}

The potential environmental savings of using a solar thermal installation in this type of heating process are compared to a solar-free installation. Three installation configurations are considered:

- No solar thermal system

- Solar thermal system (current installation)

- Solar thermal system (optimised case)

The impacts related to energy use in the form of gas and electricity as well as impacts from material components of each configuration are presented in Fig. 11.

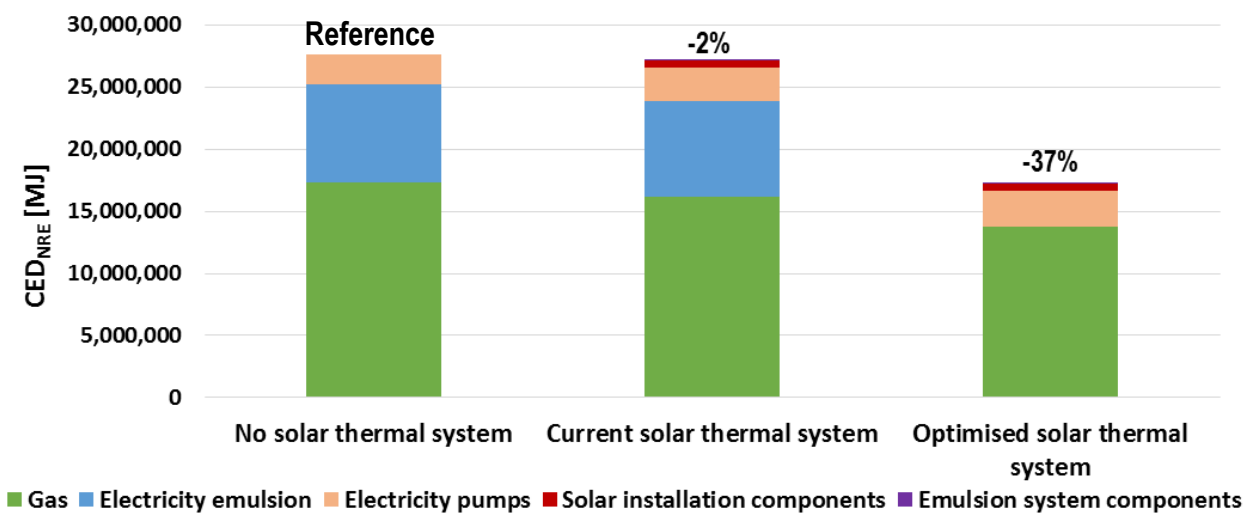

Fig. 11: Comparison of $\mathrm{CED}_{\mathrm{NRE}}$ for different configurations 
Integration of a solar thermal system in this type of industrial process potentially contributes to primary energy savings. This value is quite low for the case of an installation operated under current real conditions. The reasons are twofold: emulsion heating is primarily electric which greatly contributes to increase of the $\mathrm{CED}_{\mathrm{NRE}}$; secondly, under these conditions solar production is penalised because of the low water tank energy demand. In contrast, primary energy savings are more pronounced in the case of the optimised system since emulsion heating is now entirely supplied either with solar or gas. This configuration also results in an increase of the solar production, which directly affects gas consumption.

An environmental cost-benefit analysis showed that the energy savings brought by the solar installation could be compensated the additional material impacts within twelve years. This time is reduced to two years if the installation operates under optimal conditions, see Table 2.

Tab. 2 Payback time of the environmental cost of modifications

\begin{tabular}{|c|c|}
\hline Configuration & CED $_{\mathrm{NRE}}$ \\
\hline Current solar thermal system & $11.5 \mathrm{ans}$ \\
\hline Optimised solar thermal system & $1.2 \mathrm{ans}$ \\
\hline
\end{tabular}

\section{Conclusion}

The behaviour and performance of a medium-temperature solar industrial process for bitumen storage was investigated based on measurements and simulations for subsequent optimisation of the process. Measurements over 30 months of operation revealed a very variable consumption profile over the year, but also from one year to another. Important differences, up to $50 \%$ at $220^{\circ} \mathrm{C}$, between the certified performances of the solar thermal collectors and measured were observed during this project and consequently low specific solar production, around $100 \mathrm{kWh} / \mathrm{m} 2$. The reason for this low productivity is not only the poor performances of the collector itself, but also the non-optimised control management of the system.

Simulations show that there is room for improvement and that the solar production could be roughly doubled if the system operates as designed. Furthermore, simulations prove that the heat exchangers designed for both thermal storages are undersized. Increasing solar collector area results in higher solar productivity, but still relatively low annual collector field efficiency. Evacuated tube collectors are shown to be a cost-effective configuration for this system, but these collectors could only supply energy at low temperature level and not to the bitumen tank, as the system was designed initially.

The financial analysis shows a cost of solar energy supplied to the system at $1.80 \mathrm{CHF} / \mathrm{kWh}$. This value could be reduced to $1.18 \mathrm{CHF} / \mathrm{kWh}$ in the case of an optimised system, but it is still very high when compared to conventional energy prices. The highest share in the investment cost is not generated by the collector field, but by the system integration and mainly by the supporting structure. Therefore, with a lighter system integration and a better operation and maintenance management, the energy cost can achieve $0.47 \mathrm{CHF} / \mathrm{kWh}$, which can be acceptable for a pilot project.

The life cycle impact assessment of the solar installation showed that, as for the investment cost, the infrastructure impacts predominate over the entire life cycle, accounting for over $60 \%$ of the total $\mathrm{CED}_{\mathrm{NRE}}$. Energy savings brought along by the solar installation more than compensate the additional material and energy related impact of integrating such a system. Less than 2 years environmental return on investment periods were found in the case of an optimised solar bitumen storage system. 


\section{References}

Bunea, M., Hildbrand, C., Eicher, S., Duret, A. and Citherlet, S., 2016. Analysis of a Solar Thermal Installation for Medium Temperature Industrial Applications. Eurosun 2016 - ISES Conference Proceedings.

Eurostat. Energy, transport and environment indicators - 2016 edition

IRENA. Solar Heat for Industrial Processes. EA-ETSAP and IRENA 2015, in: Technology Brief E21.

Krummenacher, P., Muster, B., 2015. Methodologies and Software Tools for Integrating Solar Heat into Industrial Processes.

Mauthner, F., Weiss, W., Spörk-Dür, M., 2016. Solar Heat Worldwide. Markets and contribution to the Energy supply 2014 